N. E. Piskunov, W. W. Weiss, D. F. Gray, eds.

\title{
Modelling the Mass Loss of Cool AGB Stars
}

\author{
Peter Woitke \\ Zentrum für Astronomie und Astrophysik, Technische Universität \\ Berlin, Hardenbergstraße 36, Sekr. PN 8-1, D-10623 Berlin
}

\begin{abstract}
The current status of modelling the wind driving mechanism of AGB stars is reviewed. Our present understanding of these winds is substantially guided by spherically symmetric, time-dependent models which reveal a complex interplay between stellar pulsation, hydrodynamics, small-scale waves, chemistry, dust formation and radiative transfer. The level of approximation used for the physical description of the various processes, and the degree of consistence in the models are crucial and different approaches lead to rather different results. Beside reviewing the frontiers of the present theoretical research on AGB star winds, this paper presents some new results concerning the cooling of the gas behind propagating shock waves in the outer atmosphere and the self-organisation of dust-forming gases (cloud formation) caused by a radiative/thermal instability of dust forming gases.
\end{abstract}

\section{Introduction}

The overwhelming majority of all new born stars (with main-sequence mass $\approx 1 \ldots 8 M_{\odot}$ ) cease their life on the Asymptotic Giant Branch (AGB) where they attain high luminosities $\approx 3 \times 10^{3} \ldots 5 \times 10^{4} L_{\odot}$ and low effective temperatures $\approx 2000 \ldots 3200 \mathrm{~K}$ (Habing 1996, Le Bertre 1998, Wallerstein \& Knapp 1998). The winds of these cool giants are characterised by high mass loss rates $10^{-8} \ldots 10^{-4} M_{\odot} /$ yr, large dust-to-gas mass ratios of the order of $10^{-3}$ (Knapp 1985) and low outflow velocities $\approx 10 \mathrm{~km} / \mathrm{s}$. During their lifetime on the $\mathrm{AGB}$, the stars loose a substantial fraction of their mass in form of such winds on time-scales which can be shorter than typical nuclear burning time-scales (Wachter et al. 2002). Therefore, the final evolution of AGB stars is mainly mass-loss-controlled, driving them towards the planetary-nebula phase and the final cooling to white dwarfs.

AGB stars produce almost all nuclei of interstellar dust in their winds (Gehrz 1989) and the carbon stars alone return already $\approx 0.3 M_{\odot} / \mathrm{yr}$ of processed matter to the Galaxy (Wallerstein \& Knapp 1998). The winds of AGB stars are therefore essential not only to understand late stages of stellar evolution, but also the circuit of matter in the universe.

Despite its obvious relevance for the stellar evolution and the replenishment of the Interstellar Medium, the mass loss mechanism of AGB stars is still a matter of debate. In general, stellar winds are created by waves (directly or via chromospheric heating) or by radiation pressure. In the case of AGB stars, shock waves created by the stellar pulsation and condensation of dust grains close to the 


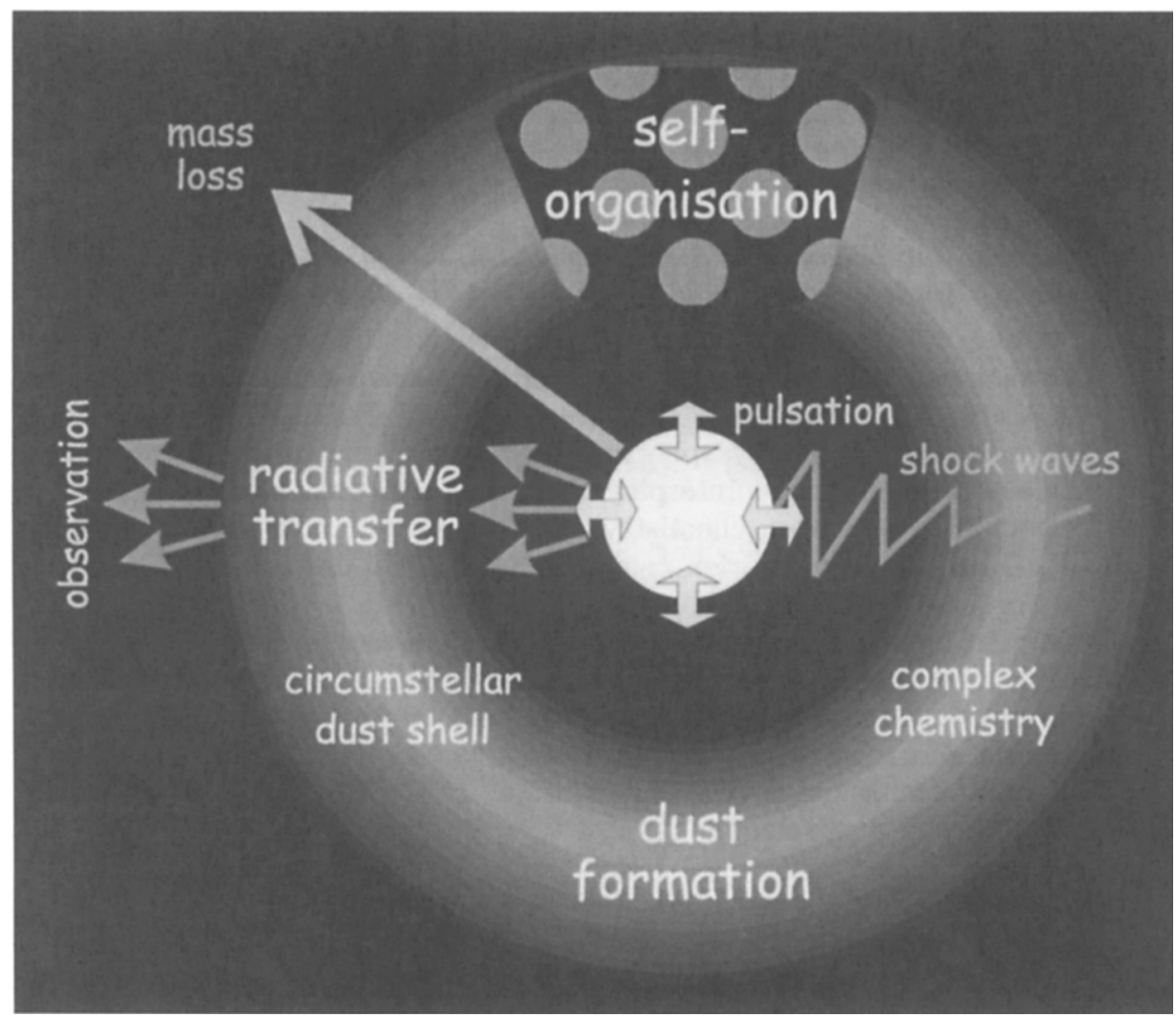

Figure 1. Sketch of the physical processes involved in the mass loss mechanism of AGB stars.

star certainly play a central role for the driving of AGB star winds as sketched in Fig. 1. However, more definite conclusions are difficult to make, because different theoretical investigations have arrived at rather different results (see Table 1). This review intends to summarise the different scenarios investigated and to discuss the physical insight provided by the various time-dependent models for the generation of the stellar winds of AGB stars, which are based on a physical description of the driving forces.

\section{Overview of AGB star mass-loss models}

The work of Wood (1979) has demonstrated that sound waves created by the stellar pulsation quickly (and inevitably!) steepen up to shock waves in the large density gradient of the stellar atmosphere. These propagating shocks can directly produce stellar outflows with very high mass loss rates $\left(\approx 10^{-2} M_{\odot} / \mathrm{yr}\right)$ if the gas in the outer atmosphere behaves adiabatically. In contrast, episodic mass loss events on a much smaller average rate $\left(\approx 10^{-12} M_{\odot} /\right.$ yr $)$ are obtained if the gas is capable to quickly radiate away the energy dissipated by the shock 
waves, i. e. when an instantaneous relaxation towards radiative equilibrium is assumed ("isothermal approximation").

Bowen (1988) has refined this model by introducing a parametric description of the radiative cooling rate of the gas which is adjusted to the non-LTE behaviour of some prominent permitted atomic lines (Willson et al. 1997). According to this model, the gas in the outer atmosphere is again and again heated by the propagating shock waves. Behind the shocks, the gas quickly cools down to $8000 \mathrm{~K}$... $10000 \mathrm{~K}$ by line emission, but then further radiative cooling becomes inefficient. Consequently, the only remaining possibility for the gas to get rid of its excess energy is to deliver work on its surroundings by adiabatic expansion. Thus, the energy input by the stellar pulsation forces the gas in the outer atmosphere to expand continuously - a pressure driven outflow heated by the pulsation is obtained. A straightforward consequence of this mass loss mechanism (pulsation + inefficient radiative cooling) is the occurrence of a region with strongly enhanced temperatures $3000 \mathrm{~K} \ldots 8000 \mathrm{~K}$ close to the star, termed as the "calorisphere" (see e.g. Fig. 2 in Willson 2000). This calorisphere is causally connected with the proposed mass loss mechanism, but probably inconsistent with many molecular observations (e.g. Reid \& Menten 1997, Hron et al. 1998). A reduction of this dissatisfying temperature increase in the outer atmosphere can be achieved by including radiation pressure on dust grains (Bowen 1988, Wood 1979), because the larger outflow velocities in this case cause a stronger adiabatic cooling. The wind is then driven by a combination of the upper mechanism and dust. However, these models rely on a very simple, additional force term in the equation of motion, parametrised as smooth step-function of temperature. The formation of the dust grains is not considered in detail.

The Berlin group around A. Fleischer, A. Gauger and J. M. Winters have improved this situation by inventing a time-dependent treatment of the dust complex, based on the assumption of equilibrium chemistry in the gas phase and the application of modified ${ }^{1}$ classical nucleation theory (Gail et al. 1984). Four additional partial differential equations in conservation form (the moment equations developed by Gail \& Sedlmayr 1988) are solved simultaneously with the hydrodynamical equations and the radiative transfer to model in detail the processes of nucleation, dust growth \& evaporation, and the element consumption (Fleischer et al. 1992, Fleischer et al. 1995). The results of the model calculations (see e.g. Fig. 5 in Winters et al. 2000) show that the dust formation process is strongly coupled to the hydrodynamics and the radiative transfer. The pulsation induced shock waves are slowly damped as they propagate through the dust-free levitated layers of the outer atmosphere of the star. When a shock wave reaches the dust formation zone close to the star, this zone is compressed which temporarily creates favourable conditions for dust formation. Radiation pressure on the newly formed dust grains accelerates and lifts the gas-dust-mixture from the levitated layers into the wind region. The levitated layers are characterised by rather simple, periodic velocity variations (positive and negative), whereas the wind region (with throughout positive velocities) often behaves chaotically.

\footnotetext{
${ }^{1}$ The concept of classical nucleation theory is utilised, but the value of the "surface tension" (an ill-defined quantity for small clusters) is adjusted to real cluster data, see Gail et al. (1984).
} 


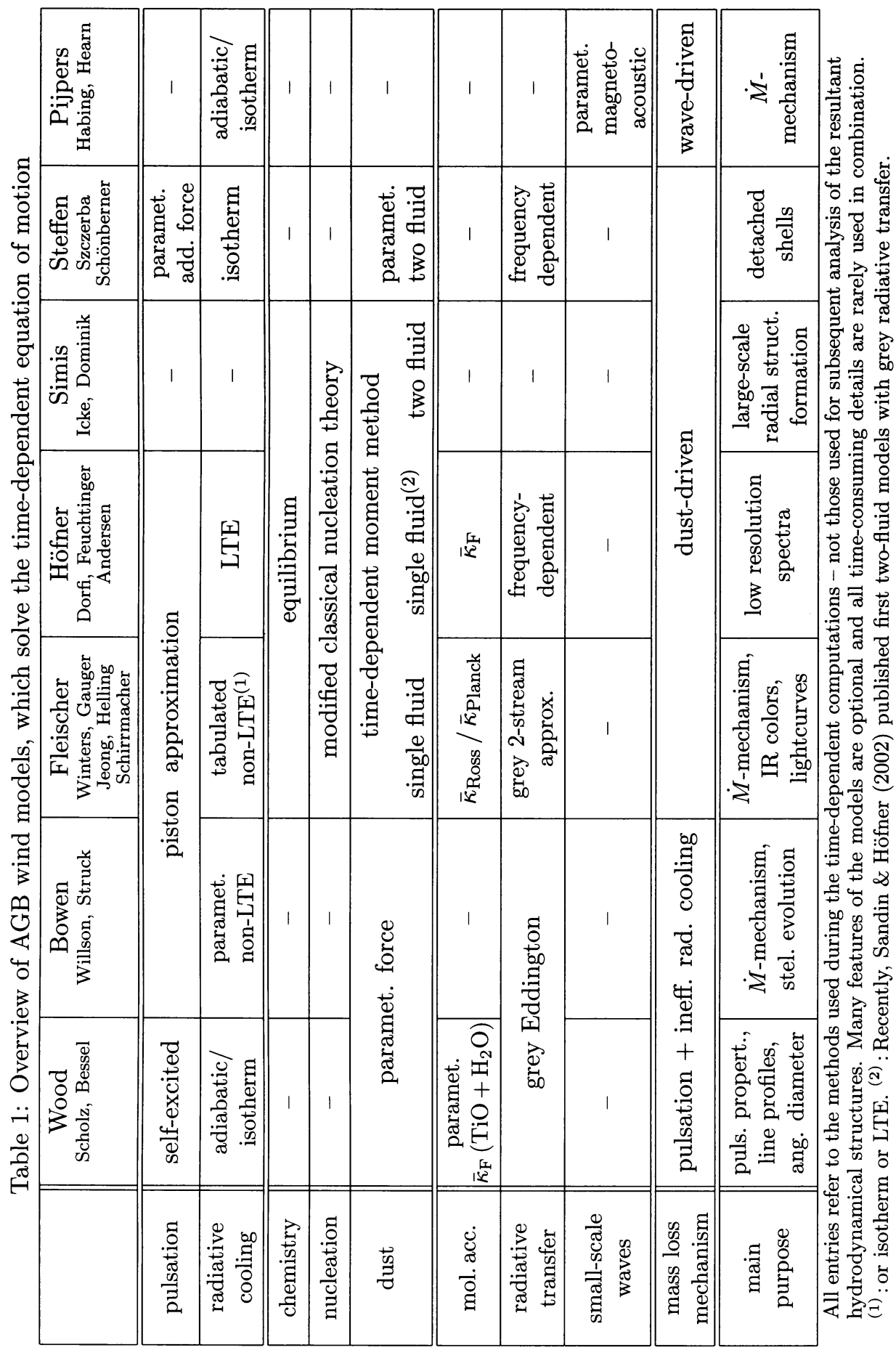


The dynamical behaviour of the dust formation zone triggered by the pulsation is the heart of the mass loss mechanism in these model calculations. According to the Berlin works, the winds of AGB stars are mainly dust-driven. The pulsation is a necessary ingredient as it temporarily creates the necessary preconditions for dust formation by increasing the gas density in comparison to static or stationary models, but pulsation alone does not lead to mass loss. As a combined effect of pulsation and time-dependent dust formation, the production of radial dust shells at a typical feature in the Berlin models, occurring in sometimes periodic, but often multi-periodic or even chaotic time intervals. Consequently, no clear correlation between the dust-to-gas ratio and the temperature exists - as used in other models. The formation of dust shells has important consequences for the lightcurves of AGB stars. The calculated monochromatic light variations show intermediate minima and long-term periods (Winters et al. 1994), i. e. features which are actually frequently observed in long-period variables. The formation of dust shells can be interpreted in terms of a dynamical instability of the dust formation zone. A compression of this zone leads to the production of more dust, which causes a hydrodynamical acceleration of the gas-dust-mixture which pushes and thereby compresses the dust-forming gas situated ahead of $\mathrm{it}^{2}$. This unstable, self-amplifying control loop leads to radial structure formation (Woitke 2001). Fleischer et al. (1995) have shown that this instability ("exterior $\kappa$-mechanism") can even lead to the production of dust shells, if the pulsation of the central star is switched off.

Höfner et al. (1995) have taken over the time-dependent description of the dust formation as described above, treating the dust-radiation-hydrodynamics with an implicit adaptive grid technique. Consequently, the conclusions concerning the mass loss mechanism and the production of dust shells are similar as those inferred from the Berlin group (see e. g. Andersen et al. 1999). However, Höfner (1999 and this volume) have recently implemented a frequency-dependent radiative transfer, utilising a sparse opacity sampling method. These new models considerably improve the physical description of the stellar atmosphere and, for the first time, seem to make possible detailed interpretations and predictions of molecular bands originating from the (levitated) stellar atmosphere and their temporal evolution (see e.g. Aringer, Nowotny, Lebzelter, Hron, this volume). Since the atmosphere is the foot-point of the wind, these models can also be expected to reveal new details concerning the wind formation in the future. Similar to the behaviour of the outer regions in line-blanketed stellar atmospheres, the wealth of molecular lines in the infrared spectral region causes a substantial lowering of the radiative equilibrium temperatures of the gas $(\approx 500 \mathrm{~K})$ in comparison to grey models, which probably allows the formation of dust to take place already much closer to the star. It must be noted, however, that a contrary effect also exists. The dust particles (with opacities typically scaling as $1 / \lambda)$ are heated up in comparison to grey models (inverse greenhouse effect) which worsens the condition for their formation.

In the models reviewed so far, the gas-dust-mixture has been treated as a single-fluid (assumption of position coupling), despite the well-known fact

\footnotetext{
${ }^{2}$ See, however, Winters et al. (2002), for a different type of results concerning slow winds with small mass loss rates and no distinct dust shells.
} 
that even supersonic drift velocities of the dust particles may occur in AGB winds. Steffen et al. (1998) were the first who published time-dependent twofluid models for dusty winds, actually combined with frequency-dependent radiative transfer. However, the model only starts around the sonic point, a simplification which does not allow for reliable conclusions concerning the mass loss rate (which is actually a parameter in this model, used to fix the permeable inner boundary condition). The models show interesting wind-wind interactions originating from different epochs of the stellar evolution, possibly related to the observations of the thin detached $\mathrm{CO}$ shells. However, the formation of dust grains has not been treated in detail. More scientific insight into the complexity of two-fluid models with dust formation has been obtained by Simis et al. (2001) who showed that two different dynamical states of the dust-gas-mixture exist. A high mass loss (high density) state, characterised by a close dynamical coupling between gas and dust with low drift velocities, and a low mass loss (low density) state, where the dust and gas components decouple dynamically and large drift velocities occur. The system may switch between these states, leading to radial shell structures on time-scales of a few hundred years.

Another principal mechanism to drive the outflows of cool giants is the direct action of magneto-hydrodynamic or acoustic waves. This scenario was considered by Pijpers \& Habing (1989) and Pijpers \& Hearn (1989). Following this completely different approach, the stellar winds can be driven by the momentum impact of small-scale waves originating from the (e. g. convective) stellar atmosphere. The basic idea is to introduce an additional force term in the equation of motion (a mean divergence of the wave pressure tensor), which expresses the time-averaged effect of small-scale waves on the dynamics ${ }^{3}$. The resulting stationary wind solutions partly agree well with the expected properties of AGB star winds (e.g. mass loss rates, final outflow velocities). Buchhammer (1994) has combined this model with time-dependent dust formation theory (as described above) in the oxygen-rich case, resulting in wave-and-dust driven winds. The obvious drawback of such models is that the physical description of the wave pressure divergence is quite rough, introducing a set of free parameters like a dissipation length and the energy of the waves at the position of the stellar atmosphere. These essential parameters can be tuned in order to get the desired results. Also, relatively large wave energies had to be considered which possibly contradicts the concept of small amplitudes (linear limit). However, it is worth to remember that these sub-scale waves - which cannot be resolved in the usually large-scale computational grids - are principally capable to drive the mass loss of cool giants, an effect which is usually disregarded by other groups.

\section{Recent developments and unsolved questions}

\subsection{Beyond piston models}

One essential shortcoming of all model calculations described so far is the lacking mutual influence between the "interior" pulsation of the star (or, generally speaking, the stellar structure) and the stellar wind. Usually, the so-called pis-

\footnotetext{
${ }^{3} \mathrm{~A}$ similar term in the energy equation, however, was not considered.
} 
ton approach ${ }^{4}$ is applied in order to concentrate on the dynamical behaviour of the outer layers responsible for the wind formation. An interesting step forward has been proposed by Bessel et al. (1996). The authors propose a two-step modelling procedure. At first, they run a non-linear pulsation code in order to calculate the dynamical behaviour of the inner layers including a radial zone just below the stellar photosphere, typically around $5000 \mathrm{~K}$. This zone serves as inner boundary condition for the second modelling step, which is a detailed dynamical model for the outer layers. According to this model, the motion of this "interface layer" for a typical Mira variable was actually found to be rather sinusoidal, but with a velocity amplitude as large as $\pm 15 \mathrm{~km} / \mathrm{s}$. Furthermore, the luminosity variation is not necessarily in phase with the motion of the interface layer and may even possess additional maxima. Unfortunately, the authors did not include any wind driving mechanism in their model (instantaneous radiative cooling is assumed and dust is neglected) such that the model does not allow for any conclusions concerning the mass loss rate.

However, a truly consistent model should, of course, contain both the interior layers responsible for the pulsation and the outer regions responsible for the wind formation, because any separation seems artificial and cannot be justified physically - a difficult task that has never been published so far. Such a model might reveal new effects, for example the formation of an optically thick dust shell might produce a strong backwarming of the stellar atmosphere and, hence, might affect the pulsation.

\subsection{Radiation pressure on molecules}

Another important question concerning the mass loss mechanism of AGB stars is the possible role of radiation pressure on molecules. Early estimates and first model calculations by Maciel $(1976,1977)$ have indicated that the photon trapping by molecules (in ro-vibrational bands, in particular by $\mathrm{H}_{2} \mathrm{O}$ ) can considerably levitate the atmosphere and drive a thin, slow outflow of very cool giants like $\mathrm{OH} / \mathrm{IR}$ stars (e.g. $T_{\text {eff }}=2000 \mathrm{~K}$ and $\log g \approx-1.5$ ). More detailed calculations (Elitzur et al. 1989, Jørgensen \& Johnson 1992) have shown that surface gravities as low as $\log g=-1.8$ are required in order to produce models where the radiative accelerations by molecules locally exceeds the stellar gravity. Similarly, Plez et al. (1992) have argued that it is probably impossible to drive an stellar outflow with molecular lines alone, at least for $\log g>-0.5$. These model calculations, however, were restricted to the static (and LTE) case, which principally underestimates the radiative acceleration by molecular lines. If hydrodynamic velocity variations with corresponding Doppler line shifts occur - as expected in pulsating atmospheres - the usual anti-correlation in wavelength space between the radiative flux and the opacity (as in the diffusion limit) breaks down, and much larger accelerations can possibly be obtained.

Following this idea, Helling et al. (2000) have studied the upper limit of the effect of radiation pressure on molecules by using Planck mean opacities in the frame of carbon-rich grey dynamical models. These models show an extremely complicated structure. Already the initial hydrostatic solution shows

\footnotetext{
${ }^{4} \mathrm{~A}$ simple sinusoidal prescription of the inner boundary condition with fixed amplitude and period, treated as free parameters.
} 

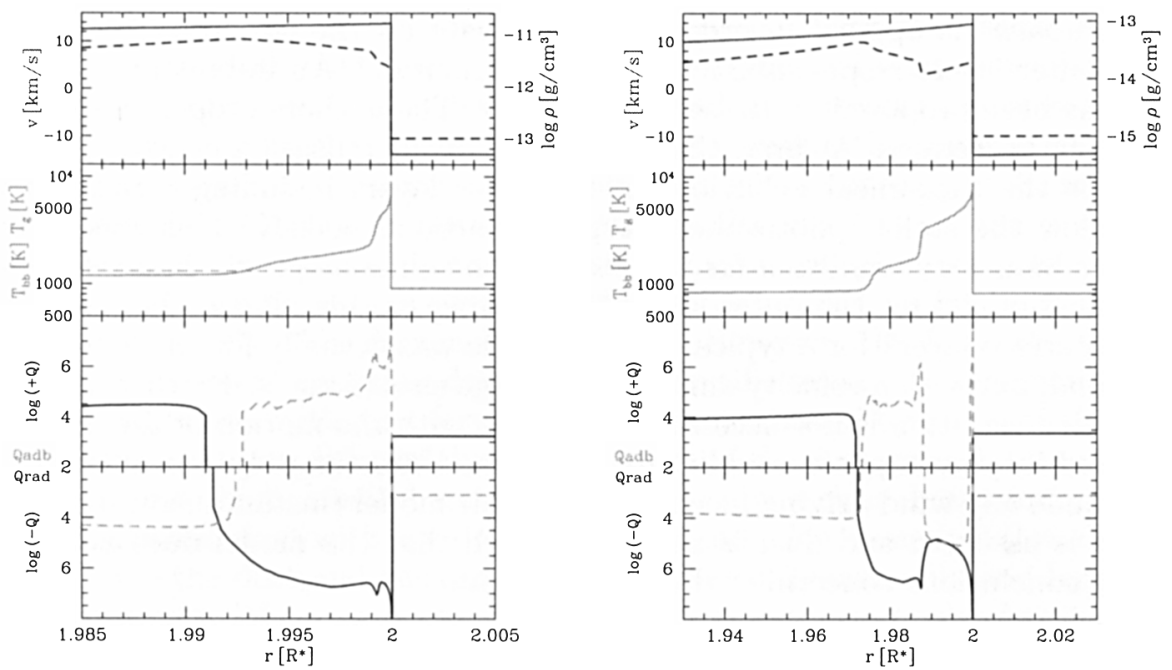

Figure 2. Radial shock wave structures calculated from periodical thermodynamical gas box calculations with detailed non-LTE radiative cooling rates. A shock wave with velocity $v_{1}=30 \mathrm{~km} / \mathrm{s}$ is considered to propagate into a gas with pre-shock density $\rho_{1}=10^{-13} \mathrm{~g} / \mathrm{cm}^{3}$ (1.h.s.) and $10^{-15} \mathrm{~g} / \mathrm{cm}^{3}$ (r.h.s.), respectively. Upper box: velocity $v$ (solid) and density $\rho$ (dashed). Middle box: gas temperature $T_{\mathrm{g}}$ (solid) and black body temperature $T_{\mathrm{bb}}$ (dashed). Lower box: Net radiative heating rate $Q_{\mathrm{rad}}$ (solid) and adiabatic work $Q_{\mathrm{adb}}=-p d V / d t$ (dashed) in $[\mathrm{erg} / \mathrm{g} / \mathrm{s}]$. Note the different scaling of the $r$-axis. Parameters: Diluted continuous radiation field with black body characteristic $T_{\text {eff }}=2600 \mathrm{~K}$ and pulsation period $P=310$ days.

a pressure-inverted region (a molecular layer swimming on the radiation field) around $2000 \mathrm{~K}$, e.g. in a model with $\log g=-0.7$, due to the combined effect of several polyatomic carbon molecules. As the temperature decreases outward, these molecules disappear and the pressure decreases again. In the dynamic case, when shock waves penetrate into this warm, opaque, pressure inverted region, the shocks are damped and partly reflected (a process which might be RichtmyerMeshkov unstable). However, the pressure inverted zone is not destroyed by the pulsation but prevailed even in the dynamic case. The model shows a rather chaotic behaviour of the dust formation zone located right above the pressure inverted region with the occasional production of dust shells connected to mass loss events - but no evidence for mass loss without dust formation.

\subsection{Shock waves and radiative cooling}

As argued in Sect. 2, the radiative cooling of the gas in the outer atmosphere is a key process to understand the mass loss mechanism of AGB stars. If the cooling is ineffective, the shock waves created by the pulsation will successively heat up the gas around the star which finally leads to a pressure-driven stellar 
outflow. If, however, the radiative cooling is fast, the gas quickly relaxes towards radiative equilibrium and the effect of pulsation-driven mass loss does not work.

In order to study the radiative relaxation behind propagating shock waves in the outer atmospheres of AGB stars, Woitke et al. (1996a,b) have developed a detailed non-LTE gas model in which all particle densities (neutral atoms, molecules, electrons and single ions) and level populations are calculated in statistical equilibrium under the influence of a continuous background radiation field. The radiative cooling rate is determined from the various free-free and bound-free transitions, atomic lines and molecular ro-vibrational transitions, including polyatomic molecules like $\mathrm{H}_{2} \mathrm{O}$ (see Schirrmacher et al. 2002). Beside a wealth of molecular lines, the model include now several thousand permitted and forbidden atomic lines, and bound-free transitions from excited levels.

Figure 2 shows two calculated radial structures of a $v_{1}=30 \mathrm{~km} / \mathrm{s}$ shock wave propagating into pre-shock densities of $10^{-13}$ and $10^{-15} \mathrm{~g} \mathrm{~cm}^{-3}$ at $r=2 R_{\star}$, respectively. The radiative cooling directly behind the shock is very effective, mainly due to hydrogen b-f transitions, Ly $\alpha$ and $\mathrm{H} \alpha$, but also due to other permitted lines like $\mathrm{Ca}$ II $\mathrm{H}+\mathrm{K}$ and $\mathrm{Mg}$ II $\mathrm{h}+\mathrm{k}$. This leads to a quick temperature decrease down to about $5000-6000 \mathrm{~K}$ within a very narrow region behind the shock (not resolved in Fig. 2). At the end of this first phase of strong radiative cooling (which also causes a post-shock compression), the permitted lines can no longer be excited collisionally and the radiative cooling becomes less effective. However, some low-lying energy levels of certain forbidden and fine-structure lines, in particular of Fe I and Fe II, can still be excited and so the radiative cooling continues slowly (Woitke \& Sedlmayr 1999), eventually assisted by adiabatic cooling in the re-expanding flow. Soon, temperatures appropriate for molecule formation are reached. Once the first molecules are present in the gas phase, the large amount of permitted ro-vibrational lines of polar molecules, in particular of $\mathrm{CO}$, causes a substantial re-increase of the radiative cooling rate which finally results in a fast relaxation towards radiative equilibrium (RE) and further post-shock compression. For the rest of the cycle, the gas remains close to $\mathrm{RE}$. The RE temperature in the molecular environment is, however, remarkably lower than the grey RE temperature $T_{\mathrm{bb}}$ as depicted in Figure 2.

This model has recently been applied to the analysis of permitted and forbidden FeII emission lines observed in Mira stars (Richter \& Wood 2001, Richter et al. 2002). The non-LTE line transfer calculations performed on the basis of these shock structures indicate are the narrow but hot post-shock cooling zone is sufficient to explain the Fe II emission line fluxes observed. In contrast, much broader hot post-shock regions would result in too strong emission lines and would contradict the observations.

The radial extent of the total radiative relaxation zone in Figure 2 amounts to $\sim 0.01 R_{\star}$ and $\sim 0.03 R_{\star}$ for pre-shock densities $10^{-13} \mathrm{~g} \mathrm{~cm}^{-3}$ and $10^{-15} \mathrm{~g} \mathrm{~cm}^{-3}$, respectively - a consequence of the general trend of decreasing radiative cooling rates per mass with decreasing densities. For even smaller pre-shock gas densities $\rho_{1} \lesssim 3 \cdot 10^{-17} \mathrm{~g} \mathrm{~cm}^{-3}$, the relaxation towards RE remains incomplete, i. e. the gas cannot radiate away the excess energy dissipated by a shock wave within one period, before the next shock encounters the gas element. Only in such a case, the occurrence of a "calorisphere" (Bowen 1988) can be expected. However, the gas density must be very small for this case to happen 
close to the star, which is only compatible with very small mass loss rates. This finding implies that the pulsation-driven mass loss as proposed by Bowen (1988) is incompatible with realistic radiative cooling rates $^{5}$. Recent model calculations of Schirrmacher et al. (2002), using the same non-LTE heating/cooling rates described above in a tabulated form, come to the same conclusion that pulsation alone cannot create the mass loss of AGB stars.

\subsection{Instabilities and structure formation}

Departures from spherical symmetry in AGB star winds are evident from high angular resolution observations like speckle interferometry, maser studies and optical observations of their successors, post-AGB stars and planetary nebulae. These observations raise important questions about the stability of the dynamical and chemical processes in the winds of AGB stars. Regarding the highly non-linear couplings and the various thermo-kinetical feedbacks already identified in these winds (e.g. the mechanisms leading to the formation of dust shells described in Sect. 2), it seems likely that instabilities of various kinds (dynamical / thermal / radiative) exist which can change the qualitative behaviour of the gas-dust-mixture in the wind generation zone quite substantially, e. g. by breaking the spherical symmetry in these winds.

One of these instabilities in dust-forming gases has recently been identified by Woitke et al. (2000). This thermal/radiative instability arises from the casting of shadows by already condensed and hence optically thick zones which improves the conditions for subsequent dust formation in the shielded, cooler regions. In order to simulate this radiative instability and to study related structure formation processes in C-star star envelopes, we have developed an axisymmetric, static model which combines equilibrium chemistry, modified classical nucleation theory and time-dependent dust growth (as described in Sect. 2) with frequency-dependent radiative transfer by means of a Monte-Carlo method (Niccolini et al. 2002).

The results of this model calculation ${ }^{6}$ are depicted in Fig. 3. The denser regions close to the star begin to condense first. Consequently, the spatial dust distribution at first resembles the slightly inhomogeneous gas density distribution assumed (see l.h.s. of Fig. 3) with a cutoff at the inner edge as a consequence of the temperatures being too high for nucleation close to the star. The process of dust formation continues for a while in this way, until the first zones become optically thick. Each already optically thick zone casts a shadow into the circumstellar envelope where the temperatures decrease by several $100 \mathrm{~K}$, thus improving the conditions for further dust formation therein. At the same time, scattering and re-emission from the already condensed zones intensifies the radiation field in regions between the optically thick zones. The stellar flux now escapes preferably through these optically thin segments, thereby heating them up and worsening the conditions for dust formation there. Thus, radially

\footnotetext{
${ }^{5}$ It has been argued that the non-LTE cooling rates described above may be incomplete (e.g. missing atomic lines) or that the applied escape probability method (Sobolev theory) might overestimate the line trapping effect (Willson 2000). Both points may be true, but would lead to even stronger cooling rates and even narrower post-shock relaxation zones.
}

${ }^{6}$ See also mpeg-movies at http://astro.physik.tu-berlin.de/ woitke/sfb555.html 

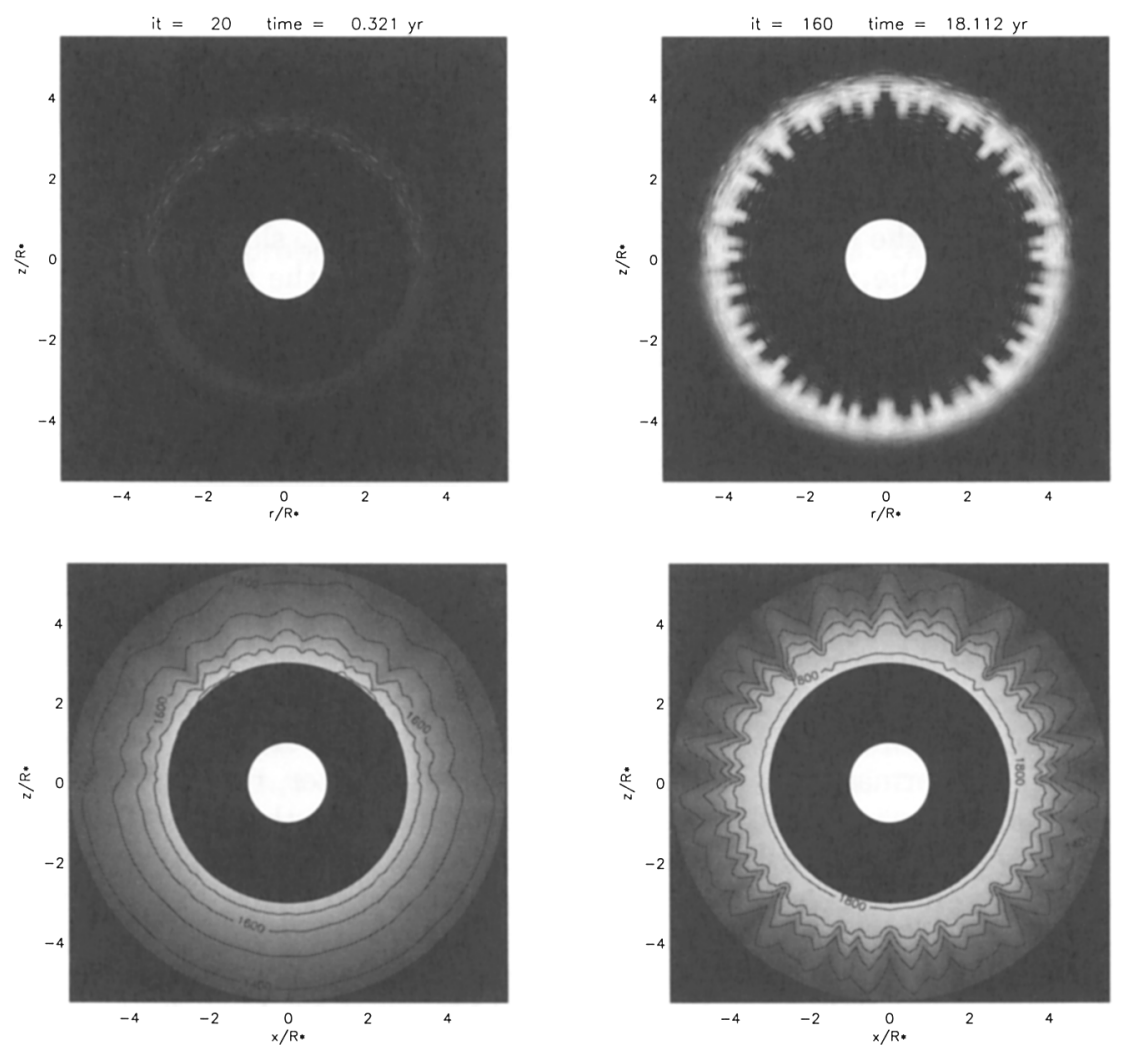

Figure 3. Self-organisation and spatio-temporal structure formation, triggered by radiative instabilities, during the formation of a dust shell around a carbon-rich AGB star. The figures show the dust temperature structure (lower row) and the logarithmic degree of condensation $\log \left(f_{\text {cond }}\right)=-3.5 \ldots 0$ (upper row) as contour plots in the $x / z$-plane at an early (l.h.s.) and a late phase (r.h.s.) as indicated on top. In the upper row, black means negligible condensation, white complete condensation. The white circles in the centre of each figure mark the star. Black regions are not included in the model. A time-independent, slightly inhomogeneous density distribution is considered. In the upper half of the model $(z>0)$, the scatter in $\rho$ is 0.15 dex, whereas the scatter in $\rho$ is 0.05 dex in the lower half of the model. Parameters: $T_{\text {eff }}=3600 \mathrm{~K}$ and $\mathrm{C} / \mathrm{O}=2$. 
aligned, cool, linear dust structures result, oriented according to the preferred direction of the radiation field and surrounded by warmer almost dust-free gas.

\section{Conclusions and outlook}

Our understanding of the physical mechanisms which drive the winds of AGB stars is still incomplete. Four major mechanisms have been proposed

1) Pulsation + inefficient radiative cooling: This mechanism works for most choices of the stellar parameters, resulting in hot, slow winds. However, regarding the wealth of molecular observations, the resulting wind temperatures seem too high. Furthermore, the dynamical calculations are not consistent with realistic radiative cooling rates.

2) Radiation pressure on dust grains seems to provide the most efficient mechanism and the various model calculations generally agree well with many observations (e. g. mass loss rates, temperatures, final outflow velocities, optical depths). It must be noted, however, that dust-driven models including a consistent treatment of the dust formation process do not exist for high effective temperatures, say $T_{\text {eff }} \gtrsim 3200 \mathrm{~K}$, and the concept of dustdriven winds may not be applicable on the complete AGB. In any case, for average AGB stellar parameters, the dust-driving apparently needs the assistance of one of the other mechanisms in this list in order to supply the dust formation zone with enough stellar matter, typically resulting in a two-step wind formation process. Furthermore, the model calculations are generally restricted to $\mathrm{C}$-stars. There are many open questions concerning the details of the chemical processes resulting in the formation of dust grains especially in the oxygen-rich case ${ }^{7}$. Moreover, oxygen-rich dust grains are generally much less effective absorbers and it is difficult to outrange the stellar gravity of a typical Mira variable by radiation pressure on such grains, even if their formation is effective. Accordingly, first consistent dynamical model calculations with time-dependent dust formation theory in the oxygen-rich case (Jeong 2000) had to consider rather extreme stellar parameters (i. e. low $T_{\text {eff }}$, large luminosities).

3) Radiation pressure on molecules: Previous attempts to explain the mass loss of AGB stars by molecular line acceleration have been successful only for very extreme stellar parameters (perhaps appropriate for OH/IR stars), i. e. in regions of the HRD where other mechanisms (e.g. dust) probably work effective anyway. For typical AGB stellar parameters, even the consideration of Planck mean opacities for the calculation of the radiative acceleration in the equation of motion (upper limit case) does not lead to a stellar outflow (Helling et al. 2000). More detailed dynamical calculations, however, which account for velocity gradients and non-LTE effects, have not been carried out so far.

\footnotetext{
${ }^{7}$ Many of the refinements in dust formation theory, e.g. kinetical approaches for the nucleation or the calculation of the radical site coverage of the dust surfaces to improve the description of growth, tend to slow down the dust formation process.
} 
4) Small-scale magneto-acoustic waves: The model calculations by Pijpers et al. have demonstrated that sub-grid waves may influence the dynamics, and are principally capable to drive moderate stellar outflows of cool stars, also with regard to early $\mathrm{M}$-type and $\mathrm{K}$-giants, i. e. where other mechanisms are problematic. However, since these model calculations rely on a very simple concept with a number of free parameters, final conclusions are difficult to made at this point.

Regarding the upper list, a combination of several mechanisms seems to provide the currently most probable explanation of AGB star winds. However, it is very seldom in physics that more than two processes of completely different nature actually attain the same level of relevance at the same time.

In order to improve our understanding of the mass loss phenomenon of AGB stars, we must improve the physical description in particular with regard to the region around $2 R_{\star}$, where the winds are usually generated.

On the one hand, this statement concerns the level of approximation used for the description of the various basic processes in the models, in particular with regard to the radiative transfer, the chemistry, the dust formation, the influence of small-scale waves/turbulence and the radiative cooling in connection with shock waves. Especially interesting was a time-dependent treatment of the $\mathrm{H}_{2}$ formation, which has an important impact of the energy budget and the chemical composition of the gas and is probably kinetically coupled with the dust formation process, and other basic improvements in dust formation theory. Another major point was to revisit the effects of sub-scale processes (turbulence/waves) which cannot be resolved in the typically large-scale computer models and are usually ignored $^{8}$. A suitable method is to include additional terms in the equations in order to model such effects, as usually done in the turbulence business or in technical hydrodynamics - a theoretical challenge.

On the other hand, the upper statement also concerns the degree of consistence in the models. A simultaneous treatment of all important physical processes is essential in such highly complex systems to identify the main controlling mechanisms. Simplifying restrictions can easily miss the important feedbacks - a numerical challenge.

Last but not least, instabilities of various types (dynamical, thermal, radiative) probably occur in these highly complex dust-forming winds. Such instabilities can provoke large-scale structure formation processes and can change the qualitative behaviour of the AGB star winds quite substantially. Regarding this possibility and the ever increasing resolving power of modern and future observational instruments, multi-dimensional models may be required.

Acknowledgments. This work has been supported by the $D F G$, Sonderforschungsbereich 555, Teilprojekt B8. I thank V. Schirrmacher for his remarks and corrections.

\footnotetext{
${ }^{8}$ Helling et al. (2001, and this volume) have e.g. studied the influence of turbulence on the dust formation process.
} 


\section{References}

Andersen, A., Loidl, R., \& Höfner, S. 1999, A\&A, 349, 243

Bessell, M. S., Scholz, M., \& Wood, P. R. 1996, A\&A, 307, 481

Bowen, G. H. 1988, ApJ, 329, 299

Buchhammer, J. 1994, Master's thesis, Technische Universität, Berlin, Germany

Elitzur, M., Brown, J. A., \& Johnson, H. R. 1989, ApJ, 341, L95

Fleischer, A. J., Gauger, A., \& Sedlmayr, E. 1992, A\&A, 266, 321

Fleischer, A. J., Gauger, A., \& Sedlmayr, E. 1995, A\&A, 297, 543

Gail, H.-P., Keller, R., \& Sedlmayr, E. 1984, A\&A, 133, 320

Gail, H.-P. \& Sedlmayr, E. 1988, A\&A, 206, 153

Gehrz, R. D. 1989, in Interstellar Dust, ed. L. J. Allamandola \& A. G. G. M.

Tielens (Dordrecht: Kluwer Academic Publishers), 445-453

Habing, H. 1996, A\&AR, 7, 97

Helling, Ch., Oevermann, M., Lüttke, M., et al. 2001, A\&A, 376, 194

Helling, Ch., Winters, J., \& Sedlmayr, E. 2000, A\&A, 358, 651

Höfner, S. 1999, A\&A, 346, L9

Höfner, S., Feuchtinger, M. U., \& Dorfi, E. A. 1995, A\&A, 297, 815

Hron, J., Loidl, R., Höfner, S., Jørgensen, U., Aringer, B. 1998, A\&A, 335, L69

Jeong, K. S. 2000, PhD thesis, Technische Universität, Berlin, Germany

Jørgensen, U. G. \& Johnson, H. R. 1992, A\&A, 265, 168

Knapp, G. R. 1985, ApJ, 293, 273

Le Bertre, T. 1997, Lecture Notes in Physics, Vol. 497, eds. J.-P. De Greve, R. Blomme, \& H. Hensberge, Springer, 133-157

Maciel, W. J. 1976, A\&A, 48, 27

Maciel, W. J. 1977, A\&A, 57, 273

Niccolini, G., Woitke, P., \& Lopez, B. 2002, A\&A, submitted

Pijpers, F. P. \& Habing, H. J. 1989, A\&A, 215, 334

Pijpers, F. P. \& Hearn, A. G. 1989, A\&A, 209, 198

Plez, B., Brett, J. M., \& Nordlund, A. 1992, A\&A, 256, 551

Reid, M. J. \& Menten, K. M. 1997, ApJ, 476, 327

Richter, H. \& Wood, P. R. 2001, A\&A, 369, 1027

Richter, H., Wood, P. R., Woitke, P., \& Sedlmayr, E. 2002, A\&A, submitted

Sandin, C. \& Höfner, S. 2002, A\&A, accepted

Schirrmacher, V., Woitke, P., \& Sedlmayr, E. 2002, A\&A, in preparation

Simis, Y. J. W., Icke, V., \& Dominik, C. 2001, A\&A, 371, 205

Steffen, M., Szczerba, P., \& Schönberner, D. 1998, A\&A, 337, 149

Wachter, A. C., Schröder, K.-P., Winters, J. M., Arndt, T. U., \& Sedlmayr, E. 2002, A\&A, 384, 452

Wallerstein, G. \& Knapp, G. R. 1998, ARAA, 36, 369

Willson, L. A. 2000, ARA\&A, 38, 573 
Willson, L. A., Struck, C., \& Bowen, G. H. 1997, in Cosmic Winds and the Heliosphere, ed. J. Jokipii, C. P. Sonett, \& M. S. Giampapa (University of Arizona Press), 155-196

Winters, J. M., Fleischer, A. J., Gauger, A., \& Sedlmayr, E. 1994, A\&A, 290, 623

Winters, J. M., Le Bertre, T., Jeong, K. S., Helling, Ch., \& Sedlmayr, E. 2000, A\&A, 361, 641

Winters, J. M., Le Bertre, T., Nyman, L.-A., Omont, A., \& Jeong, K. S. 2002, A\&A, 388, 609

Woitke, P. 2001, Reviews in Modern Astronomy, 14, 185

Woitke, P., Krüger, D., \& Sedlmayr, E. 1996 a, A\&A, 311, 927

Woitke, P., Goeres, A., \& Sedlmayr, E. 1996 b, A\&A, 313, 217

Woitke, P. \& Sedlmayr, E. 1999, A\&A, 347, 617

Woitke, P., Sedlmayr, E., \& Lopez, B. 2000, A\&A, 358, 665

Wood, P. R. 1979, ApJ, 227, 220 